\title{
Uso da câmara de ar em cirurgia ortopédica: aplicações, vantagens e desvantagens
}

\author{
The use of inner tube in orthopaedic surgery: applications, \\ advantages and disadvantages
}

\section{Uso de la cámara de aire en cirugía ortopédica: aplicaciones, ventajas y desventajas}

\author{
Jose Carlos Barbl Goncalves' \\ Yvens Barbosa Fernandes² \\ Rafael Barreto Silva ${ }^{3}$ \\ Fernando Cipoli ${ }^{3}$
}

\section{RESUMO}

Objetivo: o trabalho visou à difundir o uso da câmara de ar, de forma sistemática, em cirurgias ortopédicas, como suporte e protetor das saliências ósseas, em especial nas cirurgias de longa duração. Métodos: trata-se de um estudo prospectivo não randomizado, realizado entre 2002 e 2007, totalizando de 264 procedimentos ortopédicos. Resultados: verificou-se que, independente do tempo de cirurgia, não houve áreas de pressão nas proeminências ósseas ou lesões de nervos periféricos no pós-operatório. Conclusão: os resultados do presente estudo permitiram recomendar a câmara de ar como uma excelente opção de suporte em cirurgias ortopédicas, pois se trata de um dispositivo de baixo custo, fácil obtenção e alta reprodutibilidade.

\begin{abstract}
Objective: this paper aimed at promoting the use of the inner tube as a systematic device in orthopedic surgery, such as body holder and protector of bony projections, especially in long-term spine surgeries. Methods: this was a prospective non-randomized study, carried out from 2002 to 2007, with 264 orthopedic procedures. Results: we found that, regardless the time of surgery, there were no areas of pressure around bony prominences or injury of peripheral nerves in the postoperative follow-up. Conclusion: those results could recommend the inner tube as an excellent option like a frame in orthopedic surgery. It is an inexpensive device, easy to obtain, and without known complications.
\end{abstract}

\section{RESUMEN}

Objetivo: el trabajo tiene como objetivo difundir el uso de la cámara de aire de modo usual en cirugías ortopédicas, como el apoyo y la protección ósea de las proyecciones, en especial en la cirugía de larga duración. Métodos: este es un estudio prospectivo, no aleatorizado, realizado durante 2002 a 2007 con un total de 264 procedimientos ortopédicos. Resultados: fue encontrado que, independiente del momento de la cirugía, no hay zonas de presión sobre prominencias óseas o lesiones nerviosas periféricas en el postoperatorio. Conclusión: los presentes resultados permiten recomendar el interior del tubo como una excelente opción para el apoyo en cirugía ortopédica, ya que es un dispositivo de bajo costo, fácil de obtener y de alta reproducibilidad.
DESCRITORES: Procedimentos ortopédicos/instrumentação; Coluna vertebral/cirurgia
KEYWORDS: Orthopedic procedures/instrumentation; Spine/surgery
DESCRIPTORES: Procedimientos ortopédicos/instrumentación; Columna vertebral/cirugía

\footnotetext{
Trabalho realizado no Hospital Centro Médico de Campinas - Campinas (SP), Brasil.

1 Doutor; Assistente do Departamento de Ortopedia do Centro Médico de Campinas - Campinas (SP), Brasil.

${ }^{2}$ Assistente do Departamento de Neurocirurgia da Universidade Estadual de Campinas - UNICAMP - Campinas (SP), Brasil; Assistente do Departamento

Neurocirurgia Centro Médico de Campinas - Campinas (SP), Brasil.

${ }^{3}$ Residentes (R3) do Departamento de Ortopedia e Traumatologia do Centro Médico de Campinas - Campinas (SP), Brasil.
} 


\section{INTRODUÇÃO}

A preocupação em proteger as saliências ósseas durante a cirurgia ortopédica é uma constante, em especial em pacientes idosos, devido à maior fragilidade da pele. Em consequência, macerações da pele e lesões neurológicas periféricas são observadas após cirurgias de longa duração, como as da coluna. As lesões, em geral estão relacionadas ao posicionamento, ao formato e à superfície dos suportes utilizados. Relton e Hall desenvolveram um suporte para cirurgias espinhais que tinha por princípio diminuir a pressão abdominal e, consequentemente, o sangramento transoperatório ${ }^{1}$. Desde então, diversos autores tentam aperfeiçoar os suportes cirúrgicos, reduzindo complicações ${ }^{2}$ e facilitando a utilização da fluoroscopia ${ }^{3}$ que é de grande auxilio na identificação e localização das estruturas ósseas.

O trabalho visou a difundir a utilização da câmara de ar em cirurgias ortopédicas como uma nova opção de suporte devido às suas características, à fácil obtenção e à reprodutibilidade.

\section{MÉTODOS}

Foi realizado um estudo prospectivo e não randomizado, entre 2002 e 2007, englobando 264 procedimentos ortopédicos, em 264 pacientes. A análise da casuística foi de 113 pacientes do sexo masculino e 151 do sexo feminino (Gráfico 1).

\section{Distribuição segundo o sexo}

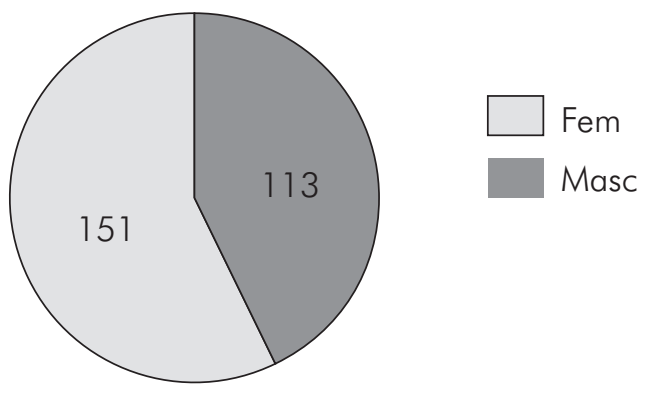

Gráfico 1

Distribuição conforme o sexo

A idade variou de 6 a 93 anos, com média de 57 anos; o tempo médio cirúrgico foi de duas horas (Gráfico 2).

Os procedimentos cirúrgicos realizados foram os seguintes: 92 vertebroplastias, 49 biópsias (13 do fêmur, 16 da coluna, 9 do ilíaco, 6 do sacro, 2 do quadril, 1 na pelve, 1 no ísquio, e 1 no acetábulo), 30 laminectomias, 39 artrodeses da coluna, 24 osteossínteses do fêmur, 17 ressecções tumorais (2 calcanectomias, 1 sacrectomia, 3 ressecções tumor paravertebral, 5 ressecções do fêmur proximal, 2 curetagem tumor supra-acetabular, 1 ressecção de tumor na coxa e 3 escapulectomias) (Gráfico 3).

Quatorze foram classificados com outros procedimentos: 2 retiradas de material de síntese, 3 descompressões da

\section{Idade ao procedimento}

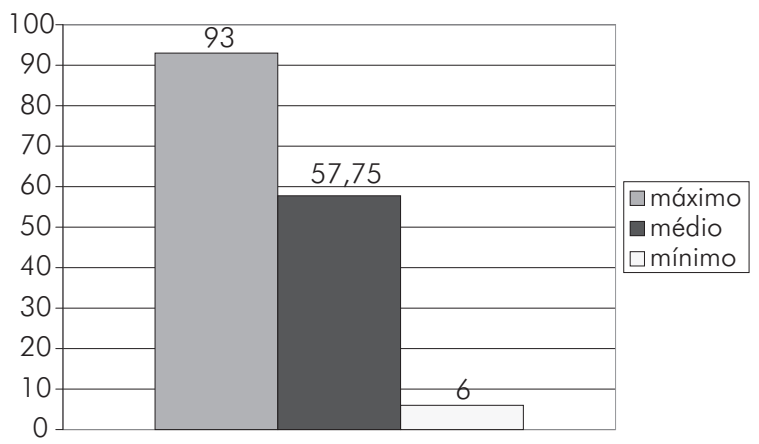

Gráfico 2

Distribuição conforme a idade

\section{Distribuição por procedimentos}

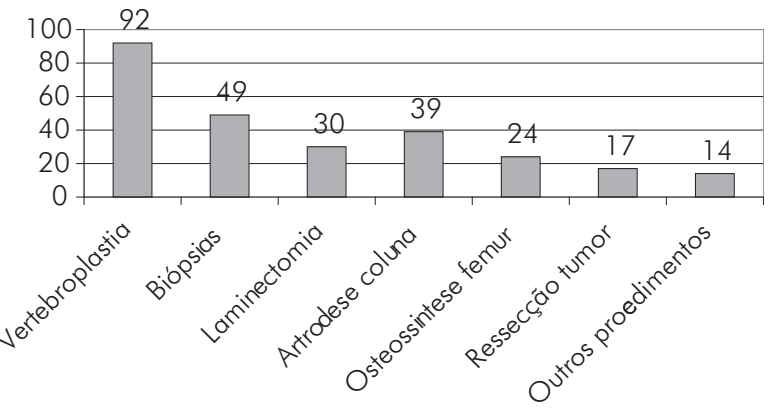

Gráfico 3

Distribuição por procedimentos

cabeça femoral, 4 cimentoplastias acetabulares, 2 suturas de tendão de Aquiles, 2 curetagens do ilíaco e uma endoprótese do fêmur proximal (Gráfico 4).

\section{Outros procedimentos}

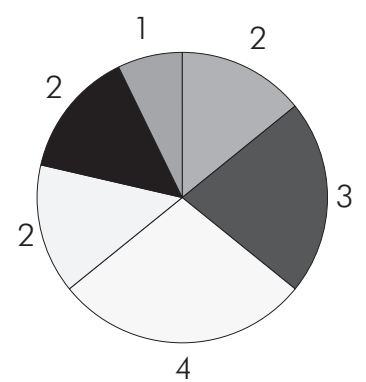

$$
\begin{aligned}
& \square \text { Ret Mat. Sintese } \\
& \square \text { Descompressão do } \\
& \text { femur } \\
& \square \text { Cimentoplastia } \\
& \text { acetábulo } \\
& \square \text { Sutura aquiles } \\
& \square \text { Curetagem ilíaco } \\
& \square \text { Endoprótese }
\end{aligned}
$$

Gráfico 4

Outros procedimentos ortopédicos

\section{Metodologia}

Utiliza-se uma câmara de ar (roda aro 13 com 30 libras de pressão) sobre a mesa de cirurgia, com cuidado para que a válvula fique direcionada para baixo e em posição que não atrapalhe a imagem da fluoroscopia (Figura 1). 


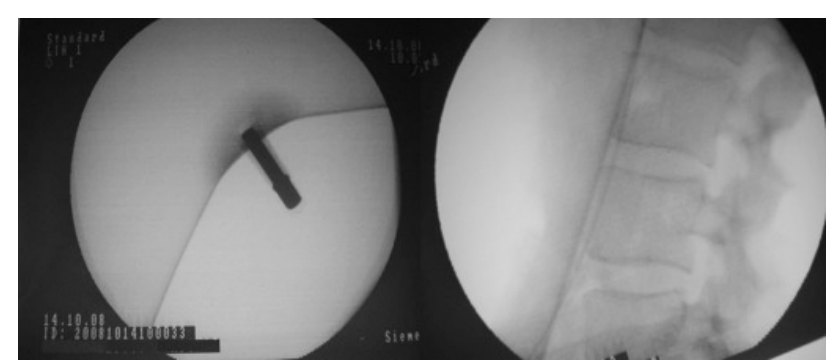

Figura 1

Bico radiopaco que deve ficar fora do campo visual e transparência da câmara na incidência em perfil da coluna

Cobre-se a câmara de ar com lençol de forma para evitar dobras e, nos pacientes longilíneos, podem ser utilizadas ataduras de crepe para reduzir o diâmetro da câmara, deixando-a oval e evitando a hiperlordose lombar. Após a anestesia, coloca-se o paciente em decúbito ventral, com apoio nas espinhas ilíacas e na região esterno peitoral, de forma a deixar livres as axilas e o abdome. O membro superior deve ser posicionado em abdução e flexão do braço, flexão do cotovelo com as mãos do paciente próximas a cabeça, tomando cuidado com a articulação glenoumeral e distensão do plexo braquial (Figura 2).

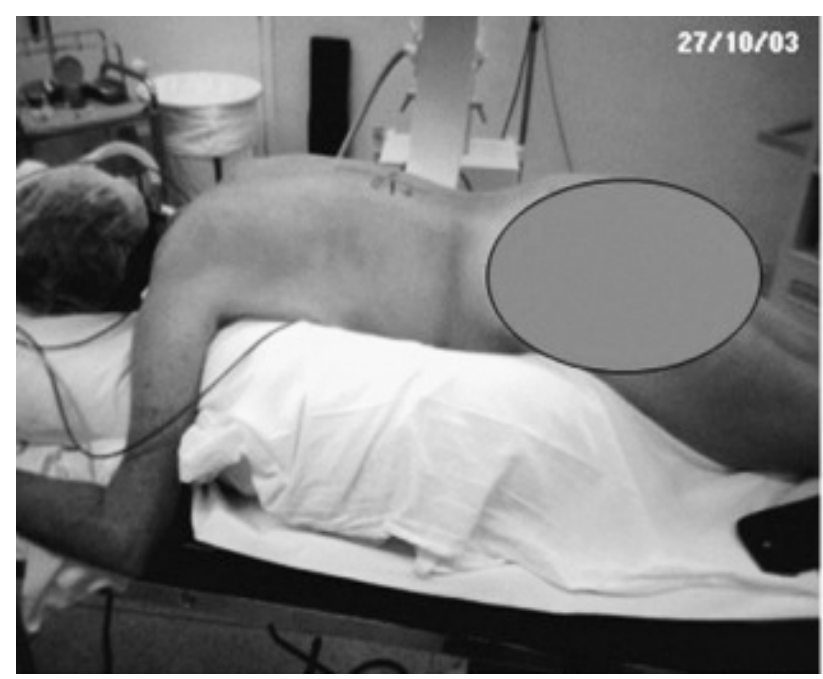

Figura 2

Câmara de ar colocada sobre a mesa operatória com paciente

Foram utilizados travesseiros para apoio da cabeça e das pernas e rolos de malha tubular para proteger a patela (Figura 3).

\section{RESULTADOS}

Nos 264 procedimentos, observou-se grande facilidade do uso da fluoroscopia para visualização das estruturas ósseas devido à radiotransparência e à ausência de artefatos metálicos (Figura 1).

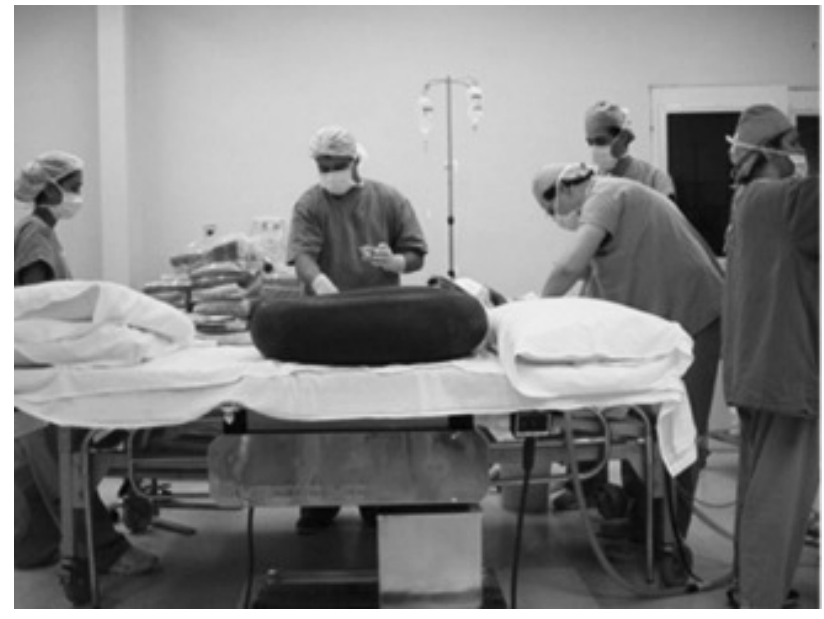

Figura 3

Câmara de ar e travesseiros para acomodação do paciente

Independentemente do tempo de cirurgia, não houve lesão neurológica periférica. Ocorreu um caso de área de hiperpressão nas proeminências ósseas no pós-operatório, decorrente da presença de dobras no lençol, o qual evoluiu bem com reperfusão da pele e sem ulcerações (Figura 4).

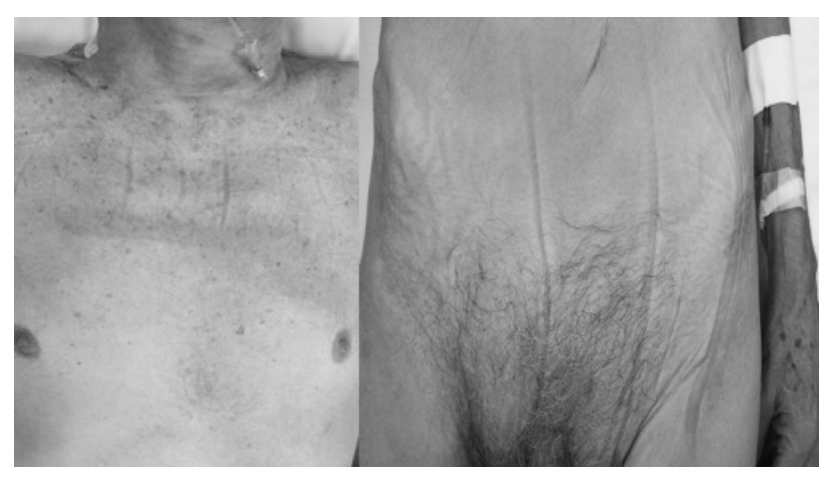

Figura 4

Áreas de hiperpressão por dobra do lençol

\section{DISCUSSÃO}

Relton e Hall desenvolveram um suporte para cirurgias espinhais que tinha por princípio diminuir a pressão abdominal e o sangramento transoperatório ${ }^{1,8}$. Desde então, diversos autores tentam aperfeiçoar os suportes cirúrgicos, reduzindo complicações ${ }^{2,4-8}$ e facilitando a utilização da fluoroscopia ${ }^{3}$, que é de grande auxílio na identificação e localização das estruturas ósseas. A transparência da câmara de ar facilita o uso do intensificador de imagem, possibilitando uma posição segura e adequada para o procedimento cirúrgico.

Coxins feitos com lençóis ou campos cirúrgicos, com apoio das proeminências ósseas, assim como mesa de apoio para cirurgias da coluna, têm sido usados com frequência. O problema desses suportes em cirurgias de longa duração é a maceração dos tecidos ao redor das proeminências 
ósseas e as complicações neurológicas periféricass, ${ }^{4,-9}$, como a compressão do nervo cutâneo lateral da coxa ao redor crista ilíaca.

O uso da câmara de ar, em comparação a coxins convencionais e mesas especiais, demonstrou um melhor acolchoamento das proeminências ósseas.

$O$ peso elevado pode ser uma das causas da compressão do nervo cutâneo lateral da coxa. Yang et al., em 2005, publicou um estudo no qual $23 \%$ dos pacientes apresentaram meralgia parestética e todos apresentavam índice de massa corpórea (IMC) maior que 23,54.

Mirosvisky et al., em 2000, relatou $20 \%$ de pacientes com meralgia parestética relacionando ao tempo cirúrgico e à compressão pela mesa de Relton e Hall ${ }^{3}$.

$\mathrm{Na}$ presente casuística, não foi evidenciado nenhum caso de compressão neurológica periférica, independente da massa corpórea e do tempo cirúrgico. Quando o paciente apresenta IMC acima de 30, aumentase a pressão da câmara de ar, para melhor acomodação do paciente.
Deixar o abdômen livre e suspenso, diminuindo a pressão abdominal, proporciona um menor sangramento em cirurgia da coluna. Rigamonte et al., em 2005, fez um estudo comparando a pressão intra-abdominal à utilização da mesa de Relton e Hall e ao suporte de Andrews, na qual não se verificou diferença significativa entre eles ${ }^{7}$.

Os suportes de Relton e Hall e o de Andrews utilizados em cirurgias de laminectomia, levam à retificação da lordose lombar, facilitando o acesso ao canal vertebral. Com o uso da câmara de ar, a posição adotada acarreta aumento da lordose lombar, com diminuição dos espaços interespinhosos, com maior dificuldade ao acesso do canal vertebral. Nesses casos, utilizou-se uma pinça de Backaus ${ }^{\circledR}$, que traciona o processo espinhoso facilitando acesso da pinça de Kerrinson ${ }^{\circledR}$, para a ressecção da lâmina vertebral.

\section{CONCLUSÃO}

A câmara de ar demonstrou ser uma excelente opção em cirurgias ortopédicas, em especial nas cirurgias da coluna vertebral. É um dispositivo prático, seguro e de baixo custo.

\section{REFERÊNCIAS}

1. Mouradian HM, Simmons EH. A frame for spinal surgery to reduce intra-abdominal pressure while continuous traction is applied. J Bone Joint Surg Am. 1977;59(8):1098-9.

2. Kumar SJ, Torres BC, Borges JL, Quinn T. A radiolucent spine frame: a modification of the Relton-Hall spine frame. J Pediatr Orthop. 1994;14(3): 383-4.

3. Mirovsky Y, Neuwirth M, Michael MD. Injuries to the lateral femoral cutaneous nerve during spine surgery. Spine (Phila Pa 1976). 2000;25(10):1266-9.

4. Yang SH, Wu CC, Chen PQ. Postoperative meralgia paresthetica after posterior spine surgery: incidence, risk factors, and clinical outcomes. Spine. 2005; 15;30(18):547-50.
5. Barclay L, Nghiem H T. Meralgia paresthetica may complicate posterior spine surgery CME. Spine. 2005;30:E547-E550.

6. Schwartz DM, Drummond DS, Hahn M, Ecker ML, Dormans JP. Prevention of positional brachial plexopathy during surgical correction of scoliosis. J Spinal Disord. 2000;13(2):178-82.

7. Rigamonti A, Gemma M, Rocca A, Messina M, Bignami E, Beretta L. Prone versus knee-chest position for microdiscectomy: a prospective randomized study of intra-abdominal pressure and intraoperative bleeding. Spine (Phila Pa 1976). 2005;30(17):1918-23.

8. Relton JE, Hall JE. An operation frame for spinal fusion. A new apparatus designed to reduce haemorrhage during operation. J Bone Joint Surg Br. 1967;49(2):327-32.
9. Gupta A, Muzumdar D, Ramani PS. Meralgia paraesthetica following lumbar spine surgery: a study in 110 consecutive surgically treated cases. Neurol India. 2004;52(1):64-6.

Correspondência
Yvens Barbosa Fernandes
Rua José Teodoro de Lima, 77/92 -
Cambuí
CEP: 13015-150 - Campinas (SP),
Brasil
Fone: (19) 3787-5000
E-mail: yvens@uol.com.br

